\title{
2 \\ Statistical Properties of Spectroscopic Binary Stars
}

Accepted by: Astrophys. Space Sci.

\begin{abstract}
As part of a study of the mass-ratio distribution of spectroscopic binary stars, the statistical properties of the systems in the Eighth Catalogue of the Orbital Elements of Spectroscopic Binary Stars, compiled by Batten et al. (1989), are investigated.

Histograms are presented of the distributions of various parameters of the systems in the Catalogue as a whole, and compared to those of the previous edition.

Histograms of binaries of various spectral types are presented. It is noticed that the early-type binaries in the Catalogue have on average higher radial-velocity amplitudes, shorter periods, and smaller semi-major axes than late-type binaries. Latetype binaries have relatively more eccentric orbits. Whether the differences noticed between the early- and late-type binaries have any significance with respect to the population of binary stars in the Galaxy is not clear, because it is very hard to assess the extent to which the Catalogue is a statistically representative and complete sample.

The distribution of semi-major axes $a_{1} \sin i$ varies considerably among different sub-samples.

The mass-ratio distribution of single-lined spectroscopic binaries is, for all (sub-)samples, characterized by a decrease in the number of systems according to a power law as $q \rightarrow 1$, for $q=M_{\text {sec }} / M_{\text {prim }}>0.25$.

The mass-ratio distribution of double-lined spectroscopic binaries (SBII) is, for all (sub-)samples but one, characterized by an increase in numbers according to a power law, as $q \rightarrow 1$. The exception to this general behaviour is the sample of SBII systems with magnitudes $m \leq 5^{\mathrm{m}}$, which has its maximum at $q \approx 0.65$.

The distributions are presented 'as they are', without corrections for selection effects.
\end{abstract}

Key words: spectroscopic binary stars - parameters - statistics - distributions 


\subsection{Introduction}

In 1989, the Dominion Astrophysical Observatory, Victoria, B.C., published the Eighth Catalogue of the Orbital Elements of Spectroscopic Binary Stars (DAO8), compiled by Batten, Fletcher and MacCarthy (1989). As part of a study of the massratio distribution of spectroscopic binary stars (1991, this thesis Chapter 3), this paper presents the statistical properties of several binary star parameters as they are derived from DAO8.

Previously, statistical data for parameters of spectroscopic binary stars have been derived by several authors from the Sixth and the Seventh versions of the Catalogue (DAO6, DAO7), which were compiled by Batten (1967), and Batten, Fletcher and Mann (1978). Results based on DAO6 and DAO7, and on supplementary observations, have been presented by (in alphabetical order): Abt and Levy $(1976,1978)$ for solar type stars and for B-type stars; Fofi et al. (1983); Garmany et al. (1980) for Otype stars; Griffin (1985); Halbwachs (1987) for main sequence systems; Jaschek and Ferrer (1972) for giants and dwarfs; Kraicheva et al. (1978); Lucy and Ricco (1979) for double-lined spectroscopic binaries; Popova et al. (1982); Staniucha (1979); Trimble $(1974,1978,1990)$; and Wolff (1978) for late-type B-stars. Reviews of existing statistical data have been presented by Abt $(1979,1983)$.

The histograms of the distributions of binary star parameters, as they are derived from DAO8, are grouped together in the figures at the end of this paper. Table 2.3, preceeding the figures, provides a legenda to the histograms.

\subsection{Purpose and scope of the present study}

This study is part of an investigation of the mass-ratio distribution of binary stars. Its purpose is primarily to investigate if the mass-ratio distribution varies among different samples of spectroscopic binary stars. To that end, distributions of the mass ratio, and of several other parameters, were derived from the Catalogue as a whole, and from various sub-samples.

The results of this study are used as an observational reference for model calculations (Hogeveen 1991, this thesis Chapter 3). In these models only systems with main-sequence components are considered. Although in the final study the models are compared to the main-sequence systems in the Catalogue, in this paper systems of all luminosity classes $(\mathrm{I}, \ldots, \mathrm{V})$ have been retained, because they provide useful information. Excluded are systems for which the spectral type designations in the Catalogue contain indications like $p$ (peculiar), $e$ (emission lines), or any other peculiarity. Sub-dwarfs are also excluded. Due to these restrictions, the study involves 655 of the 966 single-lined (SBI), and 366 of the 503 double-lined spectroscopic binaries (SBII) in the Catalogue.

The distributions are given 'as they are', without much comment. The interpretation of the distributions, and the problem of the selection effects, are the subject of separate papers. 


\subsection{Overall distributions; comparison to DAO7}

We will first consider the overall distributions of the parameters of the systems in DAO8, and compare them to the distributions derived from DAO7. Both versions of the Catalogue were available in machine-readable form.

\subsubsection{Single-lined systems}

Derived from 655 SBI systems in DAO8, Fig. 2.1 displays the histograms of radialvelocity amplitudes $K_{1}$, periods $P$, projected semi-major axes $a_{1} \sin i$, mass ratios $q$, eccentricities $e$, spectral types, and magnitudes $m$. In dashed lines, the figure displays the histograms of the same binary properties as they are derived from 480 SBI systems in DAO7. All parameters, except for the mass ratio $q$, are derived directly from the catalogues.

Mass ratios $q=M_{\text {sec }} / M_{\text {prim }}$ were calculated from the mass function, adopting an average value $\sin ^{3} i=0.679$ (Staniucha 1979), and estimating each primary mass $M_{1}$ from the spectral-type-mass relation proposed by Trimble (1974), which still compares well to more recent results published by Popper (1980) and Harmanec (1988). For the reasons explained in the previous section, of the 966 SBI systems in DAO8, only 655 are included in the statistics of Fig. 2.1, and of the 653 sBI systems in DAO7, only 480 are included.

When we compare the results derived from both versions of the Catalogue, the increase in the number of systems by about $50 \%$ in DAO 8 with respect to DAO7 is obvious in each histogram.

For radial-velocity amplitudes $K_{1}$, the general trend in the histograms is almost the same. Most notable is the increase of systems with low $K_{1}$ in DAO8, with nearly double the number of systems in the bin $0<K_{1} \leq 10 \mathrm{~km} \mathrm{~s}^{-1}$. This increase is caused by the improved sensitivity of radial-velocity measurements, which is due to diaphragm and correlation radial-velocity instruments as developed by Griffin (1967) and Baranne et al. (1979).

The histogram of periods $P$ has changed dramatically, showing an almost bimodal character in DAO8. The increase in the number of systems with long periods is almost entirely due to the observations by Griffin and others using correlation radial-velocity instruments.

Systems of low radial-velocity amplitude and long periods of course have larger semi-major axes $a$. So the changes in the histograms of $K_{1}$ and $P$ are reflected in an increase of the number of systems with larger $a_{1} \sin i$, which is seen in the histogram of $\log \left(a_{1} \sin i\right)$ derived from DAO8 with respect to the histogram from DAO7.

The changes in $K_{1}, P$, and $a_{1} \sin i$, have left the histogram of mass ratios $q$ virtually unchanged. The general trend, of a maximum at $q \approx 0.25$, and a decrease of systems according to a power law as $q \rightarrow 1$, is preserved.

The histogram of eccentricities $e$ has not changed much.

The histograms of the spectral types have changed considerably. Most notable is the increase in the number of systems of spectral types $\mathrm{O}$ and $\mathrm{B}$, and $\mathrm{G}$ and $\mathrm{K}$. The increase in the number of early-type stars is mainly due to the studies by Garmany and Conti (1980), Abt and Levy (1978), and Wolff (1978). The increase in the number 
Table 2.1: Comparison of distributions of binary-star parameters as derived from DAO7 and DAO8, by means of the Kolmogorov-Smirnov test.

\begin{tabular}{lll}
\hline & \multicolumn{2}{l}{ KS-test result } \\
\cline { 2 - 3 } Parameter & $D$ & $P_{\mathrm{KS}}$ \\
\hline Radial Velocity $K_{1}$ & 0.09 & 0.02 \\
Period $P$ & 0.11 & 0.004 \\
Semi-Major Axis $a_{1} \sin i$ & 0.1 & 0.01 \\
Mass Ratio $q$ & 0.025 & 0.99 \\
Eccentricity $e$ & 0.09 & 0.04 \\
Magnitude $m$ & 0.11 & 0.002 \\
\hline
\end{tabular}

of late-type stars is due to the use of correlation radial-velocity instruments by Griffin (1985) and others, which are particularly suited to measure radial velocities by means of metal lines, which of course mostly applies to the spectra of late-type stars.

In the histogram of the magnitudes of SBI systems most notable is the increase in numbers for $m>5^{\mathrm{m}}$. The increase is mainly due to observations of fainter early type $(\mathrm{O}$ and $\mathrm{B})$ and fainter late type $(\mathrm{G}$ and $\mathrm{K})$ systems.

In the model calculations (Hogeveen 1991, this thesis Chapter 3), distributions of binary-star parameters are compared to the distributions derived from DAO 8 by means of the Kolmogorov-Smirnov test (Sachs 1981). Table 2.1 gives the results of the KS-test when it is applied to the distributions of DAO7 and DAO8. For each parameter the table gives the KS-statistic $D=-\infty<x<\infty\left|\Phi_{\mathrm{DAO} 8}(x)-\Phi_{\mathrm{DAO} 7}(x)\right|$ (the $\Phi(x)$ indicate the cumulative probability distribution of parameter $x$ ), and the probability $P_{\mathrm{KS}}$, $\left(0<P_{\mathrm{KS}} \leq 1\right)$ that the distributions are the same. When a confidence limit of $1 \%$, or $P_{\mathrm{KS}}>0.01$ is maintained, all parameters, except for period $P$ and magnitude $m$, are accepted as being drawn from the same distribution. However, we know that the systems in DAO7 and DAO8 are drawn from the same population (= the binary systems in the Galaxy), so low probabilities $P_{\mathrm{KS}}$ must be regarded as indications of the incompleteness of DAO7, and warnings of the possible incompleteness of DAO8. These warnings add to the remarks on the completeness of our knowledge of spectroscopic binaries made by Batten and Fletcher (1989). The comparison probability $P_{\mathrm{KS}}=$ 0.99, as it is found for the mass-ratio distributions, is quite extraordinary. It indicates that the observed mass-ratio distribution is, to a large extent, independent of the other parameters.

\subsubsection{Double-lined systems}

Histograms of properties of the double-lined spectroscopic binary stars in DAO8 and DAO7 are displayed in Fig. 2.2. Involved in the statistics are 366 of the 503 SBII systems in DAO8, and 248 of the 325 SBII systems in DAO7. For these systems the spectral-type designations did not indicate any peculiarities. Histograms are displayed for radial-velocity amplitudes $K_{1}$ and $K_{2}$, periods $P$, projected semi-major axes $a_{1} \sin i$, mass ratios $q$, eccentricities $e$, spectral types of both components, and magnitudes $m$. 
All parameters, except for mass ratio $q$, were taken directly from the catalogues. The mass ratios were calculated from

$$
q=\frac{M_{2}}{M_{1}}=\frac{K_{1}}{K_{2}}
$$

Notable in all histograms is again the increase in the number of systems in DAO8, by more than $50 \%$ with respect to DAO7. The general trends of the histograms are preserved rather well.

Notable in the distribution of radial-velocity amplitudes is the increase in the number of systems with 'low' $K_{1}$. The increase in numbers is for the SBII systems also due to observations of fainter systems, as may be seen from the distribution of magnitudes.

\subsection{Sub-samples}

\subsubsection{Variable and non-variable systems}

The DAO8 contains a fair number of systems that are indicated as 'variable', which means that they are eclipsing binaries, or suspected eclipsing binaries. Eclipsing systems are seen almost edge-on $(\sin i \approx 1)$. In view of their importance for mass determinations, and also because they are easily recognised as binaries, eclipsing systems are expected to be over-represented with respect to non-eclipsing systems.

\section{Single-lined systems}

Figure 2.3 shows the histograms of $K_{1}, P, a_{1} \sin i, q, e$, spectral types, and $m$, for 112 SBI systems in DAO8 that are indicated as 'variable'. In dashed lines, Fig. 2.3 shows the histograms of the same parameters for 543 non-variable SBI systems.

Quite notable is the number of A type stars among the variable SBI systems, with respect to the sample of non-variable systems, which is dominated by spectral types $\mathrm{B}$ and $\mathrm{K}$.

There is a considerable difference between the distributions of magnitudes of variable and non-variable SBI systems. The maximum in the histogram of the nonvariable systems is in the bin $6^{\mathrm{m}}<m \leq 7^{\mathrm{m}}$, whereas for the variable systems it is in the bin $8^{\mathrm{m}}<m \leq 10^{\mathrm{m}}$. This is a clear indication of the fact that the variable stars draw extra attention to themselves, and are over-represented with respect to the non-variable systems.

\section{Double-lined systems}

Figure 2.4 shows the histograms of $K_{1}$ and $K_{2}, P, a_{1} \sin i, q, e$, spectral types, and $m$, for 148 SBII systems in DAO8 that are indicated as 'variable'. In dashed lines, Fig. 2.4 shows the histograms of the same parameters for 218 non-variable SBII systems.

In the histograms of the radial-velocity amplitudes it may be noticed that the differences in $K_{1}$ and $K_{2}$ are larger for the variable systems than they are for the non-variable systems. We will see that this is reflected in the mass-ratio distributions $\phi(q)$ observed for both groups. 
Among the variable SBII systems, there are significantly more short-period systems (with periods $P<8$ hours) than there are among the non-variable systems. This is reflected in the distribution of semi-major axes, where there are more variable SBII systems in the bin $0.5<\log \left(a_{1} \sin i\right) \leq 1$, or $3<a_{1} \sin i \leq 10 R_{\odot}$, than nonvariable systems. This may be due to the fact that close systems are more likely to be seen (partially) eclipsing at a greater range of inclinations $i$ than wide systems, with $a \gg R_{1}+R_{2}$, the sum of radii of the binary components.

Quite notable are the differences between the mass-ratio distributions of variable and non-variable SBII systems. The $q$-distribution of non-variable systems shows an increase according to a power law from $q=0.2$ to $q=1$, with a clear maximum at $q=1$. For variable systems $\phi(q)$ shows two maxima, one in the bin $0.4<q \leq 0.5$, and one in the bin $0.9<q \leq 1$.

The distributions of eccentricities $e$ of both variable and non-variable SBII systems show that most systems have circular orbits, with $e$ close to 0 . The histogram of $e$ for variable systems shows even fewer systems of $e>0$ than that of the non-variable systems. This may be due to the fact that variable SBII systems have on average shorter periods and smaller semi-major axes than the non-variable systems have.

The differences in the spectral types of variable and non-variable SBII systems are less pronounced than they are for the SBI systems.

The distributions of the magnitudes of both groups of SBII systems agree quite well with those of the SBI systems, with the maximum of the non-variable systems in the bin $6^{\mathrm{m}}<m \leq 7^{\mathrm{m}}$, and the maximum of the variable systems in the bin $8^{\mathrm{m}}<m \leq 9^{\mathrm{m}}$.

\subsubsection{Quality of the orbits}

Batten et al. (1989) have attributed quality ratings to the orbits in their Catalogue, ranging from high quality orbits, rated as $a$, to preliminary results, rated as $e$. Figures 2.5 and 2.6 represent the histograms of the parameters of, respectively, 155 SBI and 119 SBII systems in DAO8, with quality ratings $a$ or $b$. In dashed lines, the figures represent the histograms of 500 SBI and 247 SBII systems respectively, rated $c, d$, or $e$.

\section{Single-lined systems}

Radial velocities can be measured more accurately for the late-type stars with their many metal lines, than they can for the early-type stars, with their broad $\mathrm{H}$ and $\mathrm{He}$ lines. This is why we see an emphasis on late-type stars in the histograms of SBI systems with orbits rated $a$ or $b$ (Fig. 2.5, solid lines), and an emphasis on early-type stars in the histograms of systems with quality ratings $c, d$, or $e$ (Fig. 2.5, dashed lines).

The general trend in the distribution of radial velocities $K_{1}$ is the same for systems of high and low quality, although the decrease in numbers according to a power law, seen with increasing $K_{1}$, is more pronounced for the high quality systems.

The period $(P)$ distribution is rather flat for the high quality systems, whereas it is decreasing (towards longer periods) for low quality orbits. The differences found 
between the periods of high-quality systems and those of low-quality systems, are reflected in the distributions of the projected semi-major axes, which have opposite trends.

The mass-ratio $(q)$ distributions also differ; this may be attributed to the quality with which the orbits have been determined. For single-lined systems with small mass-ratios, the radial-velocity amplitudes $K_{1}$ are quite small also, as it is the less massive, unobserved component that moves faster. Small radial-velocity amplitudes are hard to measure accurately, so the quality of the orbits will deteriorate for systems with low $q$.

The maximum in the magnitude distributions is to be found in the bin $7^{\mathrm{m}}<m \leq$ $8^{\mathrm{m}}$ for the high quality systems, and in the bin $6^{\mathrm{m}}<m \leq 7^{\mathrm{m}}$ for the low quality systems. This is due to the fact that the late-type systems in DAO8, for which the orbits are on average of higher quality, are fainter than the early-type systems, which have orbits of, on average, poorer quality.

\section{Double-lined systems}

Most notable in the histograms of the SBII systems (Fig. 2.6) is the difference in the mass-ratio distributions $\phi(q)$. The $q$-distribution of the high-quality systems peaks very strongly for $q \rightarrow 1$, whereas the distribution of low-quality systems involves relatively more low $q$ systems. The difference may be accounted for by the accuracy with which the spectra of SBII systems can be measured.

Lucy and Ricco (1979) presented a formula for the visibility $f$ of the lines of the secondary component in the spectrum of a binary, with respect to the lines of the primary component:

$$
f=\frac{2}{1+q^{-6}}
$$

This formula indicates that the visibility of the lines of the secondary in a spectrum decreases rapidly as $q$ becomes smaller. So, for lower $q$, it is harder to measure the spectra accurately. This may account for the larger number of systems of lower $q$ values in the histogram of SBII systems with quality ratings $c, d$, or $e$, and the strong peak at $q=1$ in the histogram of systems rated $a$ or $b$.

\subsubsection{A magnitude-limited sub-sample}

Figure 2.7 shows the histograms of the parameters of 153 SBI (solid lines) and 58 SBII systems (dashed lines), for a sub-sample which was derived from DAO8 by selecting only the systems of magnitude $m \leq 5^{\mathrm{m}}$.

For the SBI systems in this sample, most notable is that the systems with low radial-velocity amplitudes, longer periods and larger semi-major axes, are relatively more abundant than they are in DAO8 as a whole (cf. Fig. 2.1).

For the SBII systems, most notable is the change in the distribution of mass-ratios $\phi(q)$. The distribution no longer peaks at $q=1$, but in the bin $0.6<q \leq 0.7$. Below $q=0.6$ the drop in the number of systems is in agreement with the general trend observed for all other (sub-)samples of SBII systems. 
Table 2.2: Numbers of systems involved in the histograms of mass ratios and semi-major axes per spectral type, as presented in Figs. 2.8 and 2.9.

\begin{tabular}{lcccccccc}
\hline Spectral Type & All & O & B & A & F & G & K & M \\
\hline SBI & 655 & 16 & 193 & 110 & 85 & 95 & 137 & 10 \\
SBII & 366 & 15 & 107 & 60 & 97 & 60 & 24 & 1 \\
\hline
\end{tabular}

\subsubsection{Sub-samples of various spectral types}

Figure 2.8 shows the histograms of the $q$-distributions of the SBI (solid lines) and SBII (dashed lines) systems of spectral types O, B, A, F, G, K, and M in DAO8. Figure 2.9 shows the histograms of the distributions of semi-major axes for the various spectral types. Other parameters, like the periods and the radial-velocity amplitudes, have been omitted for reasons of conciseness. Table 2.2 displays the number of systems per spectral type involved in the histograms of Figs. 2.8 and 2.9.

\section{Single-lined systems}

Among SBI systems it is noticed, that early-type systems have on average higher radial-velocity amplitudes, shorter periods, and hence smaller semi-major axes than the late-type systems.

Also the fact that the late-type systems in the Catalogue are on average of larger magnitude than the early-type systems, may be noticed. However, there are some variations and exceptions.

Variations are also exhibited by the mass-ratio distributions $\phi(q)$ among the various spectral types. But all distributions more or less comply to the general trend of a maximum at $q \approx 0.25$, and a decrease in numbers according to a power law as $q \rightarrow 1$.

\section{Double-lined systems}

Among SBII systems, there also is a trend towards low radial-velocity amplitudes, longer periods, and larger semi-major axes as the systems are of later spectral type.

The mass-ratio distributions follow a general trend of an increase in numbers according to a power law as $q \rightarrow 1$. An exception are the B-type SBII systems, which exhibit a relatively large number of lower $q$ systems. However, as we may notice from the histograms of the variable systems, quite a number of B-type systems are variable, and when they are left out, the distribution is more in agreement with $\phi(q)$ of the other spectral types.

\subsection{Conclusions}

The number of spectroscopic binary stars in DAO8 has increased by about $50 \%$ with respect to DAO7. The increase in numbers varies considerably among spectral 
types: the number of O-type binaries increased by about $300 \%$ (their actual numbers being still quite low - about 25 in the sample with no peculiarities in their spectraltype designations), B-type stars increased by about $35 \%$, and G- and K-type stars increased by about $30 \%$ and $110 \%$ respectively. The number of $\mathrm{A}$ and $\mathrm{F}$ binaries in DAO8 is almost the same as in DAO7.

The increase must be attributed to the special interest in the last decades for early-type stars (Garmany et al. 1980, Abt and Levy 1978, Wolff 1978, and many others), and to the development of new radial-velocity instruments (Griffin 1967, Baranne et al. 1979), which triggered many observations of late-type stars.

The differences between DAO8 and DAO7, and what caused them, are particularly interesting, because they indicate that the Catalogue should be regarded very cautiously when it is used as a statistical sample from which one tries to determine characteristics of binary-star parameters. The increase in numbers per spectral type, and the differences noticed between variable and non-variable spectroscopic binaries, are only indications of the severe selection effects to which the Catalogue is subject.

The difference found between the mass-ratio distributions of SBII systems in the Catalogue as a whole, and the magnitude limited sub-sample of systems with $m \leq 5^{\mathrm{m}}$, is another such indication. The mass-ratio distributions, of both SBI and SBII systems, and the selection effects that obscure them from direct determination, are the subject of a separate paper (this thesis Chapter 3). In this paper the varying distributions of semi-major axes will also be addressed.

Table 2.3: Legenda to the histograms of Figs. 2.1 through 2.7.

\begin{tabular}{ccll}
\hline Panel & Parameter & Unit & Description \\
\hline (a) & $K_{1}, K_{2}$ & $\mathrm{~km} \mathrm{~s}^{-1}$ & Radial velocity amplitude \\
(b) & $\log (P)$ & $(\log )$ Days & Period of the system \\
(c) & $\log \left(a_{1} \sin i\right)$ & $(\log ) R_{\odot}$ & Projected semi-major axis \\
(d) & $q$ & dimensionless & Mass-ratio $\left(q=M_{\text {sec }} / M_{\text {prim }}\right)$ \\
(e) & $e$ & dimensionless & Eccentricity \\
(f) & $\mathrm{Sp} 1, \mathrm{Sp}^{a}$ & no dimension & Spectral type \\
(g) & $m$ & $\mathrm{~m}$ & Magnitude of the system \\
\hline
\end{tabular}

${ }^{a}$ Spectral types are indicated by numbers along the $x$-axis; the intervals $(0,1),(1,2), \ldots$, $(6,7)$ correspond to spectral types $\mathrm{O}, \mathrm{B}, \ldots, \mathrm{M}$. 


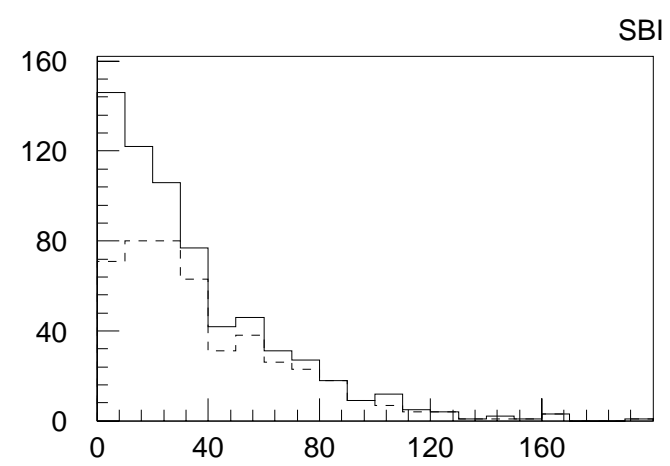

(a) $\mathrm{K} 1(\mathrm{~km} / \mathrm{sec})$

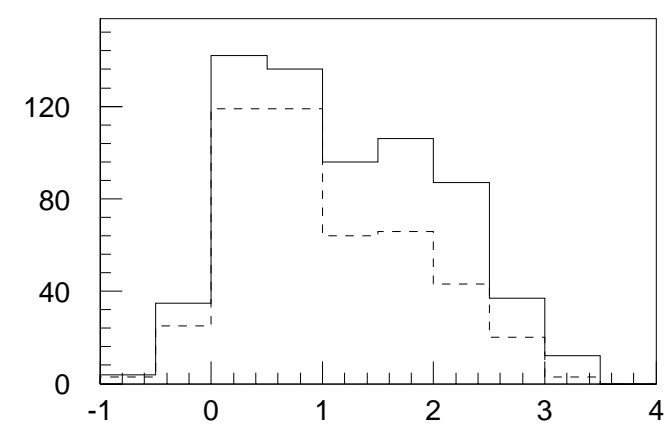

(c) Semi-Major Axis

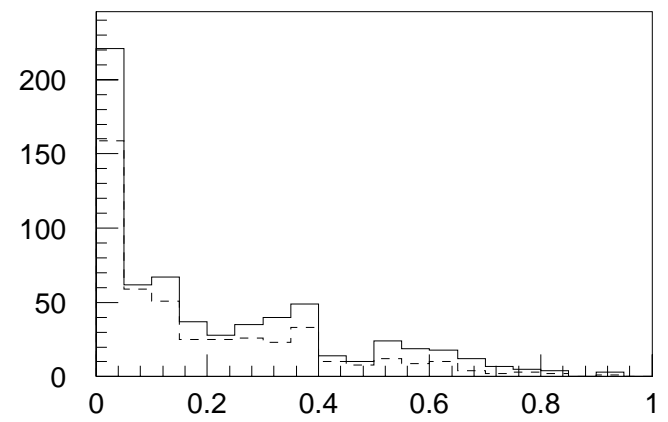

(e) Eccentricity

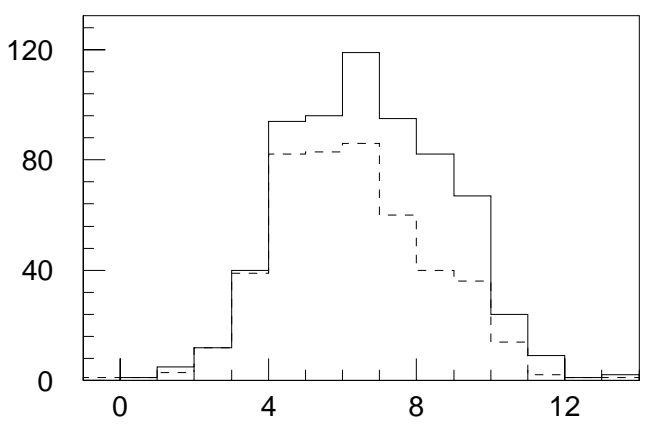

(g) Magnitude
SBI STATISTICS

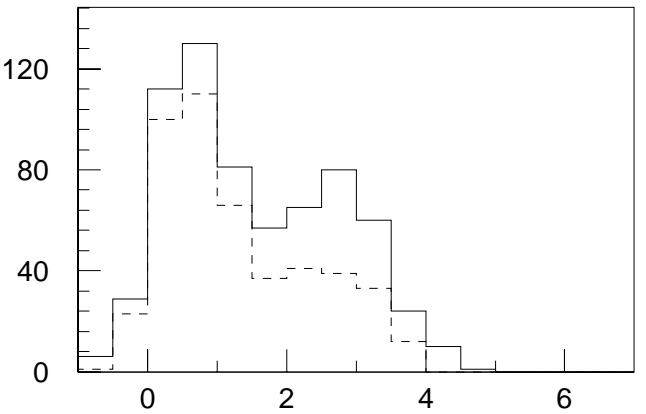

(b) Period (log days)

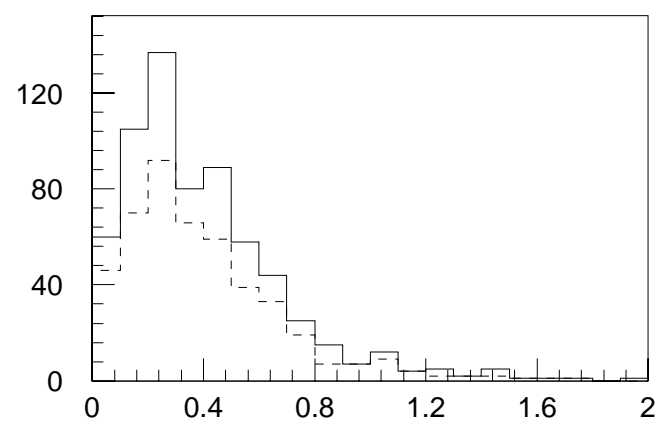

(d) Mass Ratio

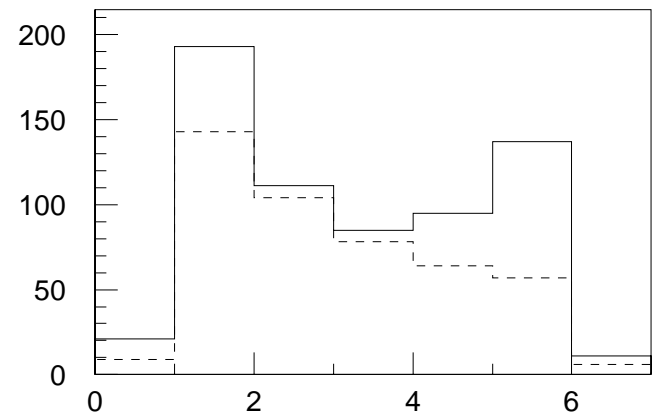

(f) Spectral Type

Figure 2.1: Solid lines: histograms of 655 SBI systems in the Eighth DAO Catalogue. Dashed lines: histograms of 480 SBI systems in the Seventh DAO Catalogue. For a legenda to the histograms, see Table 2.3. 


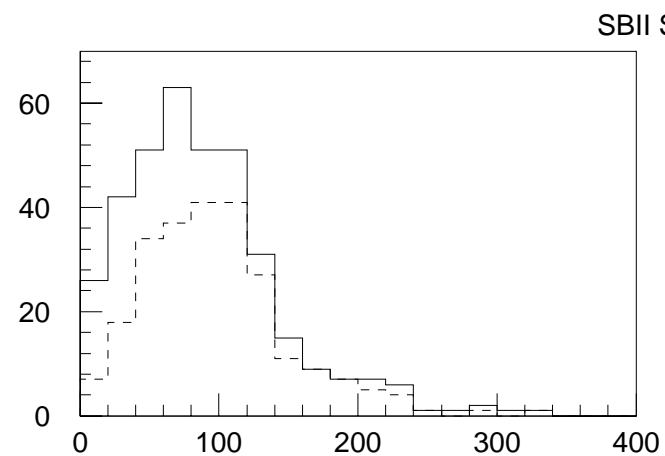

(a) $\mathrm{K} 1(\mathrm{~km} / \mathrm{sec})$

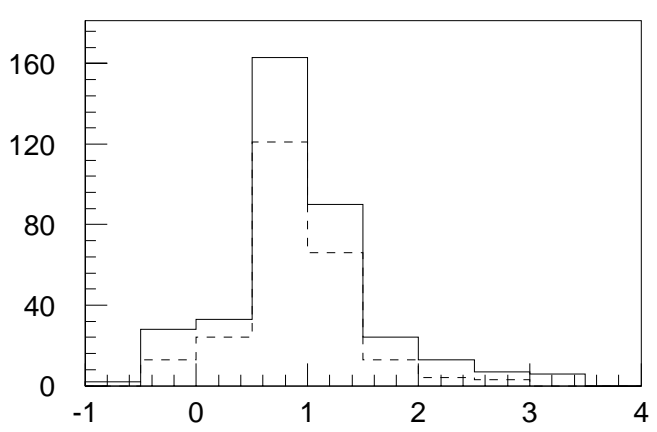

(c) Semi-Major Axis

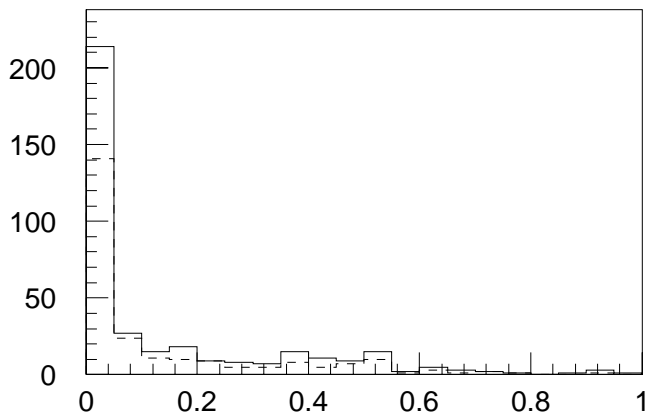

(e) Eccentricity

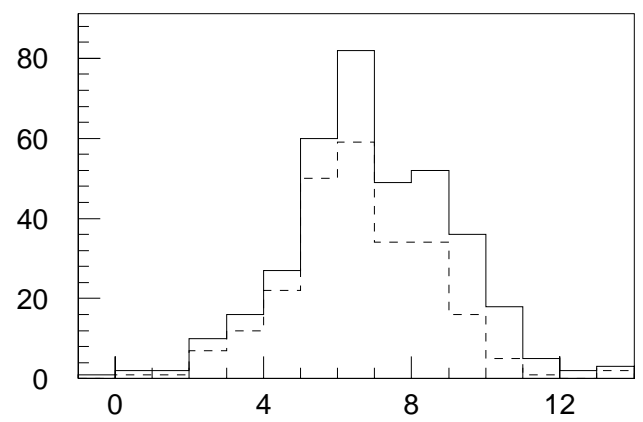

(g) Magnitude

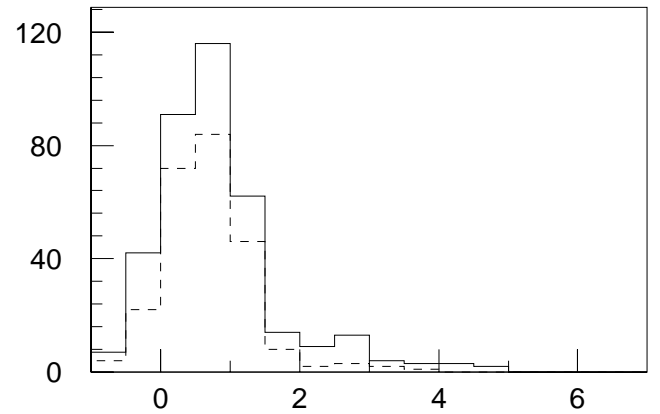

(b) Period (log days)

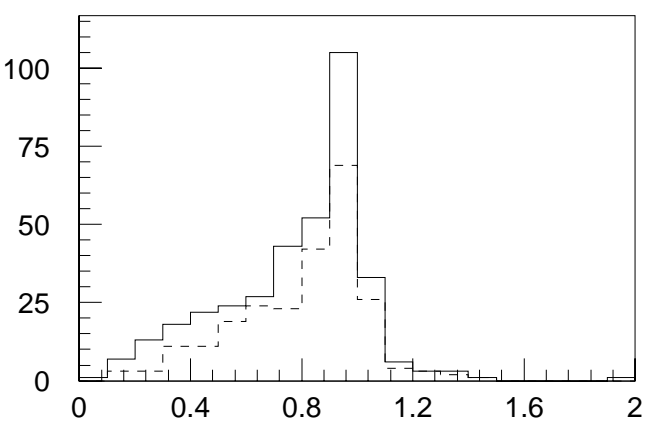

(d) Mass Ratio

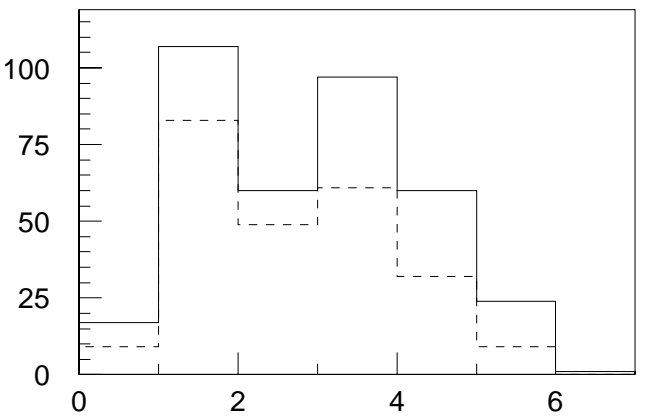

(f) Spectral Type

Figure 2.2: Solid lines: histograms of 366 SBII systems in the Eighth DAO Catalogue. Dashed lines: histograms of 248 SBII systems in the Seventh DAO Catalogue. For a legenda to the histograms, see Table 2.3. 


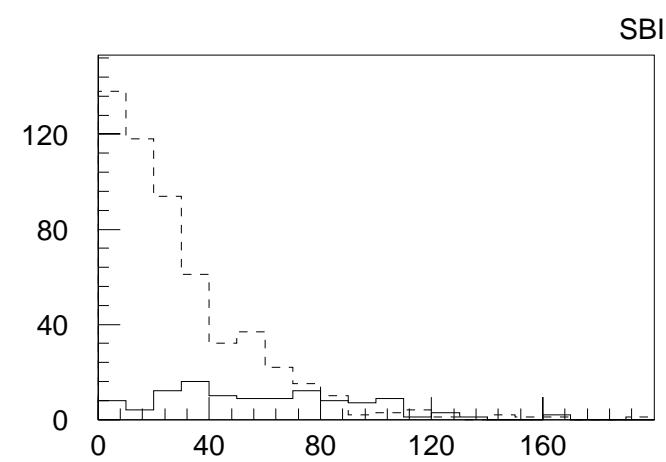

(a) $\mathrm{K} 1(\mathrm{~km} / \mathrm{sec})$

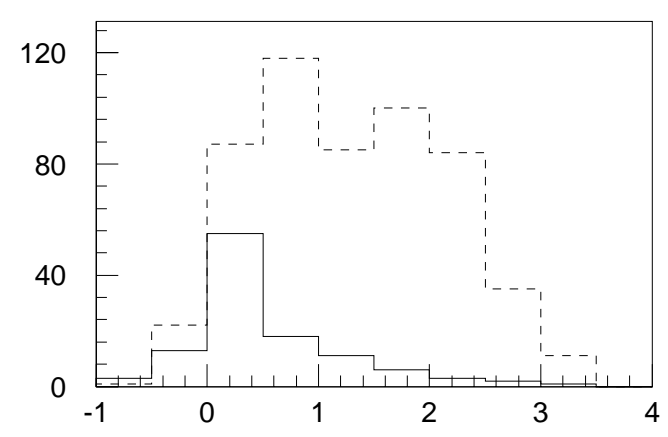

(c) Semi-Major Axis

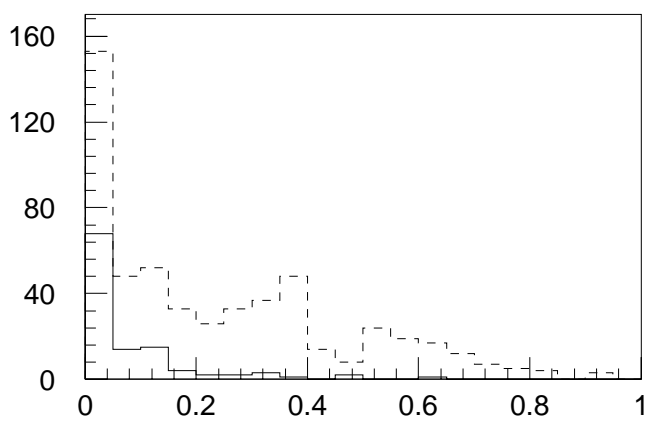

(e) Eccentricity

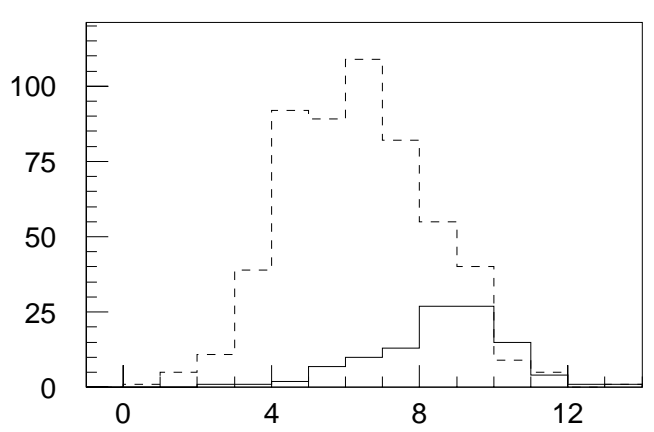

(g) Magnitude
BI STATISTICS

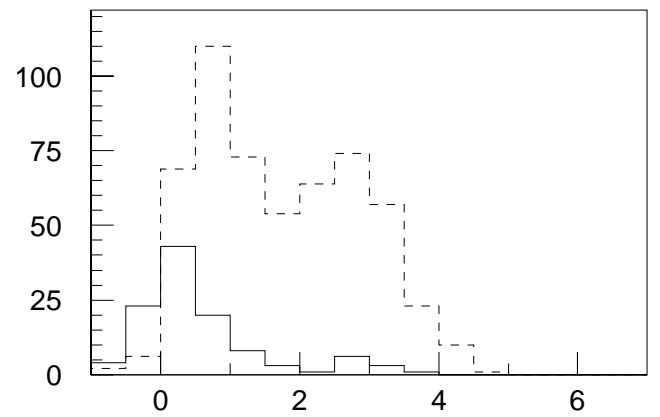

(b) Period (log days)

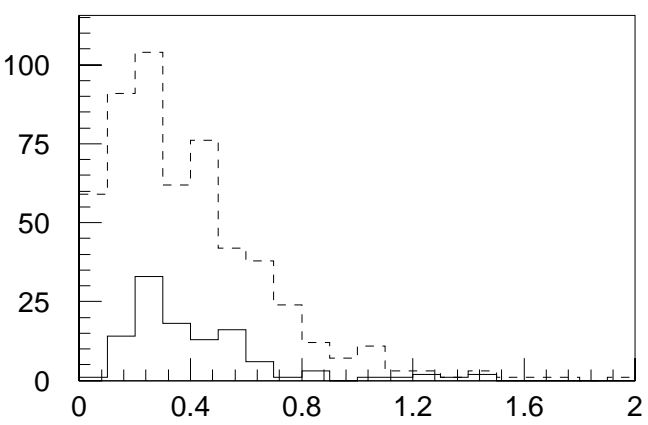

(d) Mass Ratio

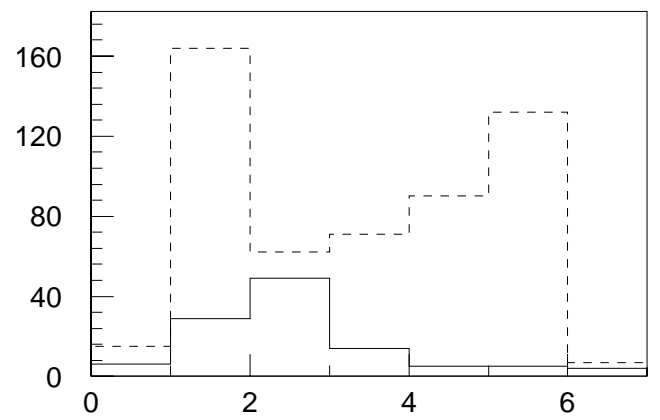

(f) Spectral Type

Figure 2.3: Histograms of 112 variable SBI systems (solid lines) and 543 non-variable SBI systems (dashed lines) in the Eighth DAO Catalogue. For a legenda to the histograms, see Table 2.3. 


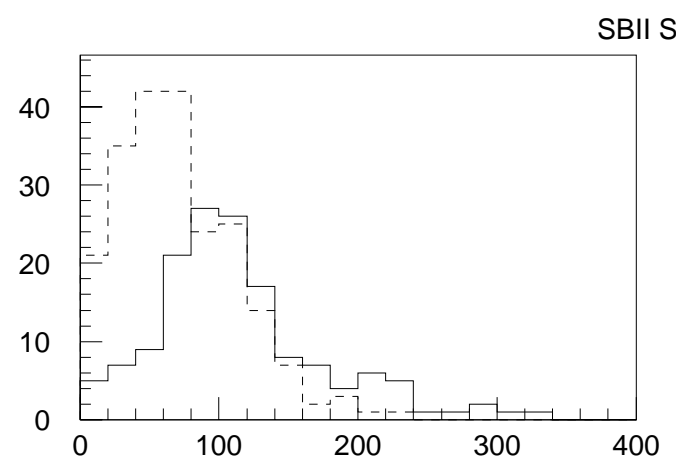

(a) $\mathrm{K} 1(\mathrm{~km} / \mathrm{sec})$

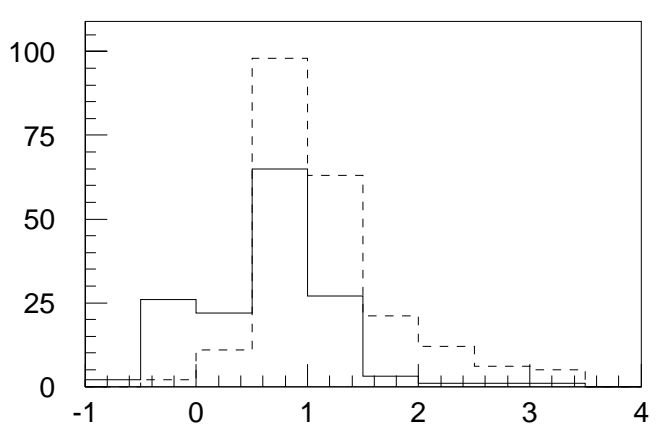

(c) Semi-Major Axis

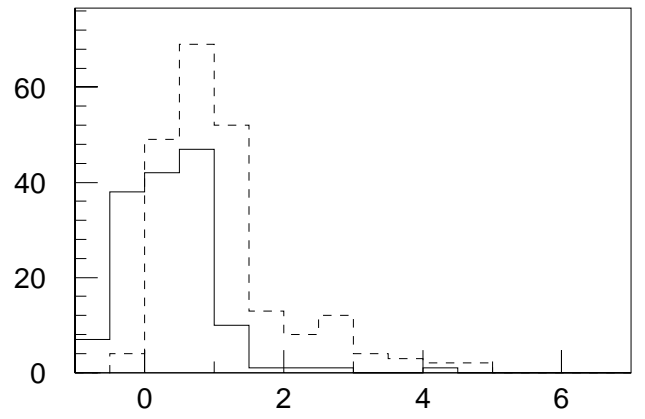

(b) Period (log days)

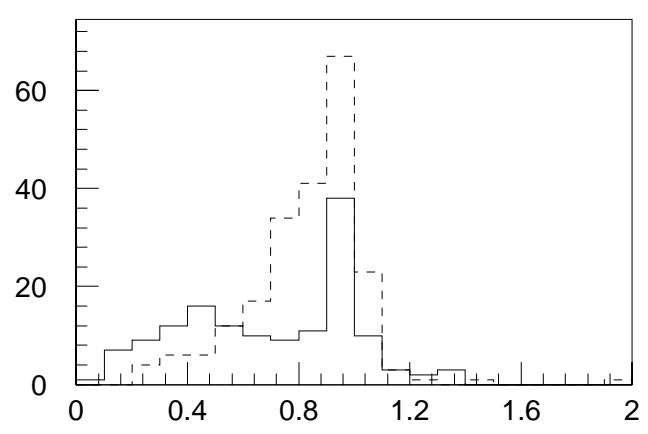

(d) Mass Ratio

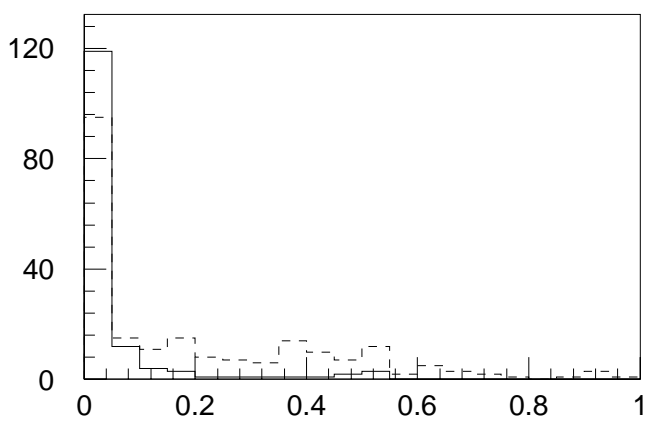

(e) Eccentricity

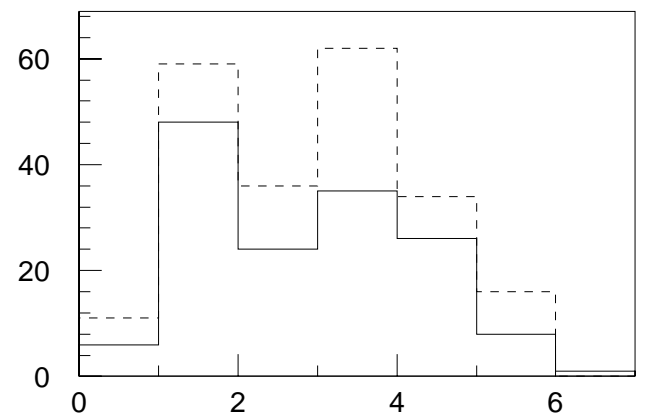

(f) Spectral Type

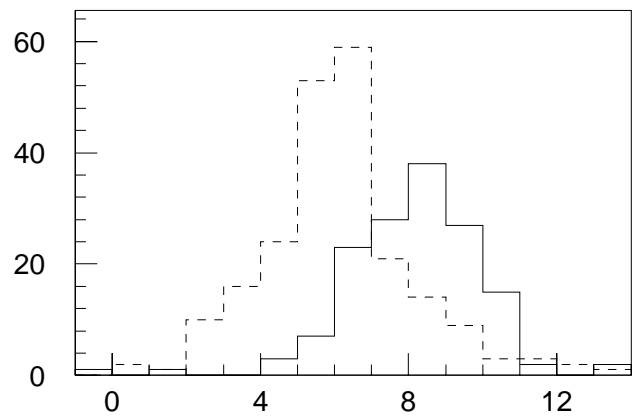

(g) Magnitude

Figure 2.4: Histograms of 148 variable SBII systems (solid lines) and 218 non-variable SBII systems (dashed lines) in the Eighth DAO Catalogue. For a legenda to the histograms, see Table 2.3. 


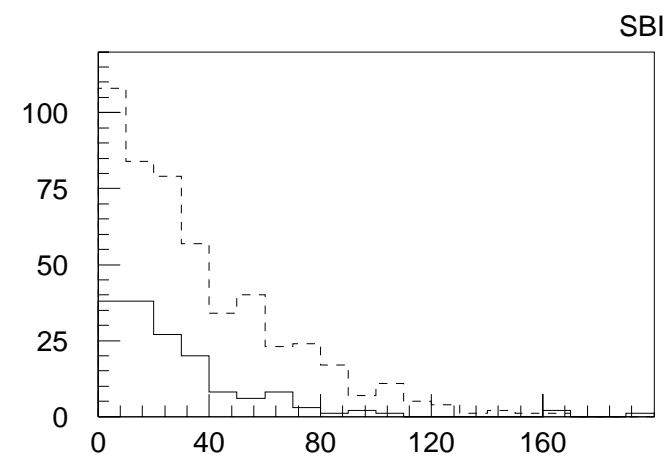

(a) $\mathrm{K} 1(\mathrm{~km} / \mathrm{sec})$

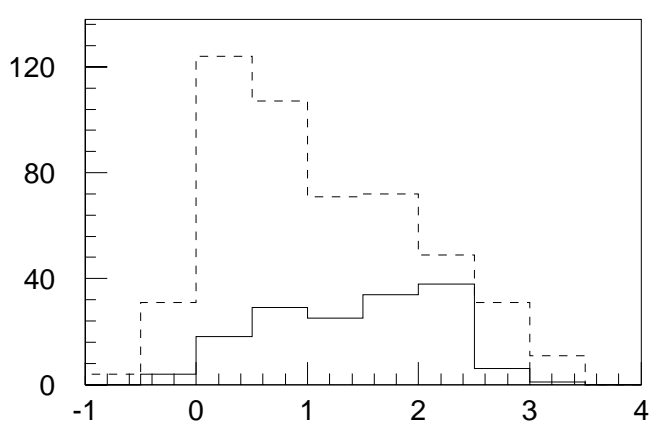

(c) Semi-Major Axis

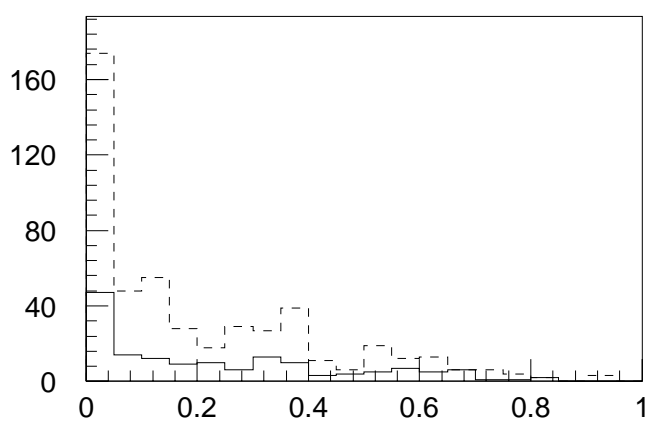

(e) Eccentricity

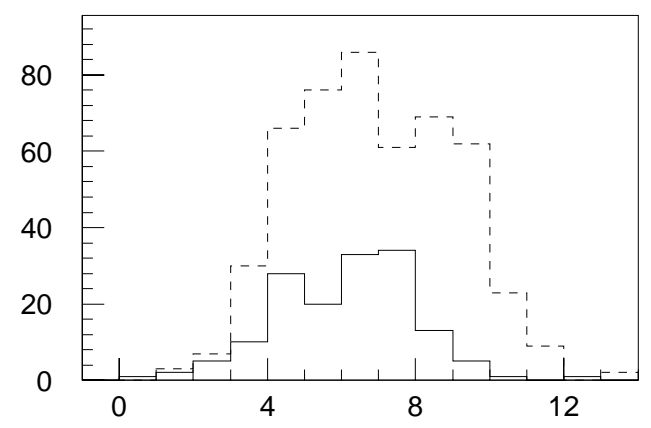

(g) Magnitude

SBI STATISTICS

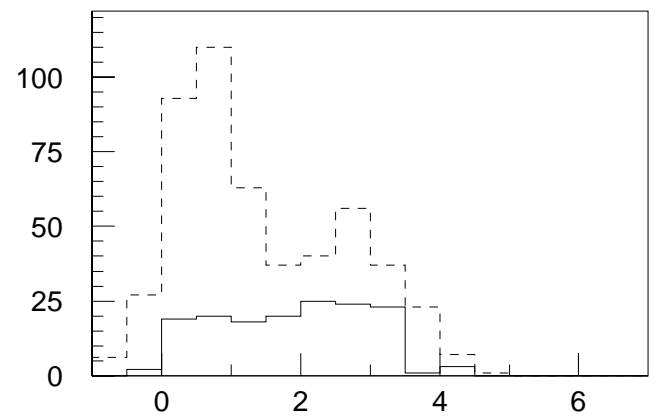

(b) Period (log days)

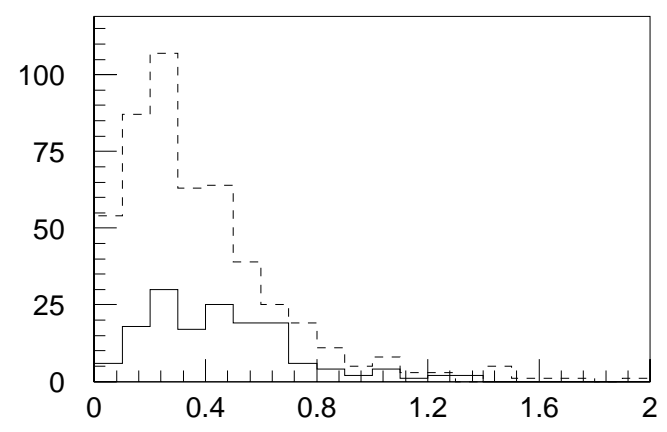

(d) Mass Ratio

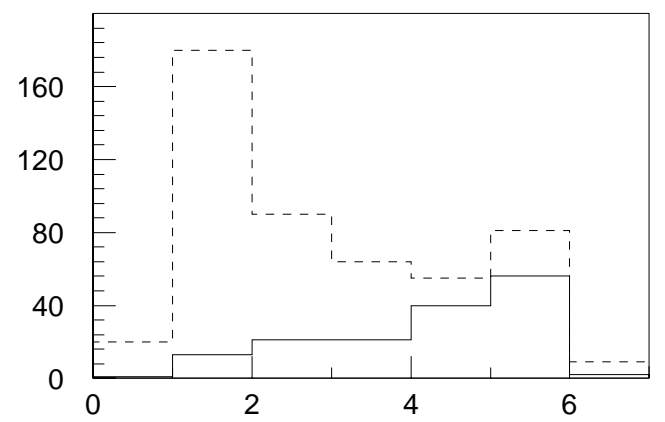

(f) Spectral Type

Figure 2.5: Histograms of 155 SBI systems in the Eighth DAO Catalogue with quality ratings $a$ or $b$ (solid lines), and 500 SBI systems with quality ratings $c, d$, or $e$ (dashed lines). For a legenda to the histograms, see Table 2.3. 


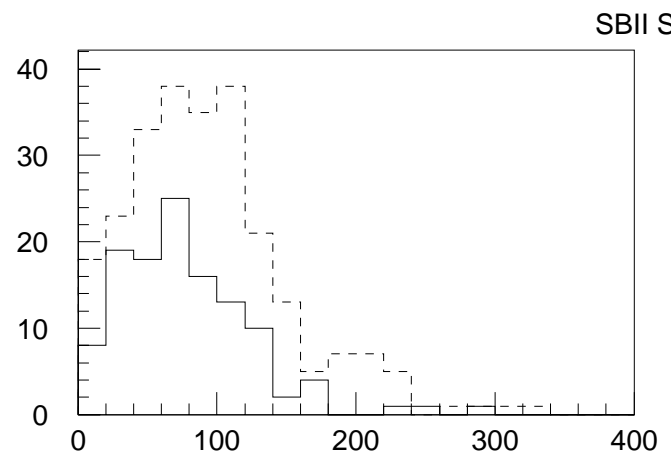

(a) $\mathrm{K} 1(\mathrm{~km} / \mathrm{sec})$

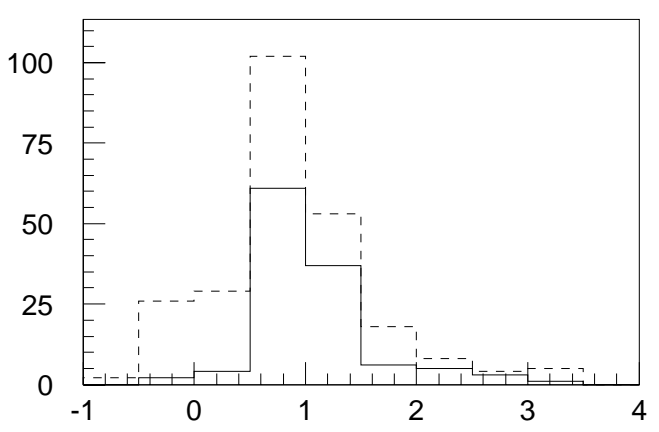

(c) Semi-Major Axis

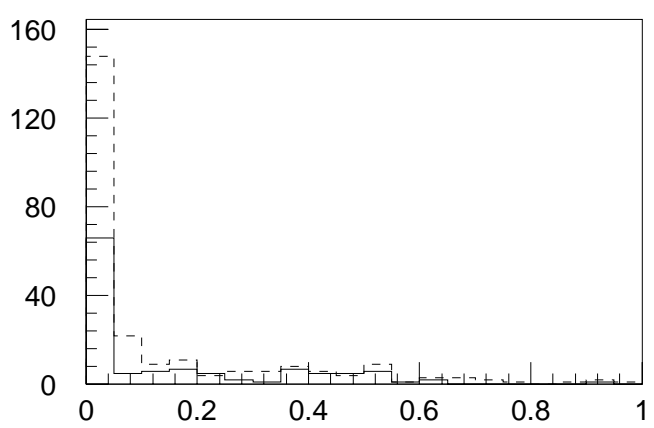

(e) Eccentricity

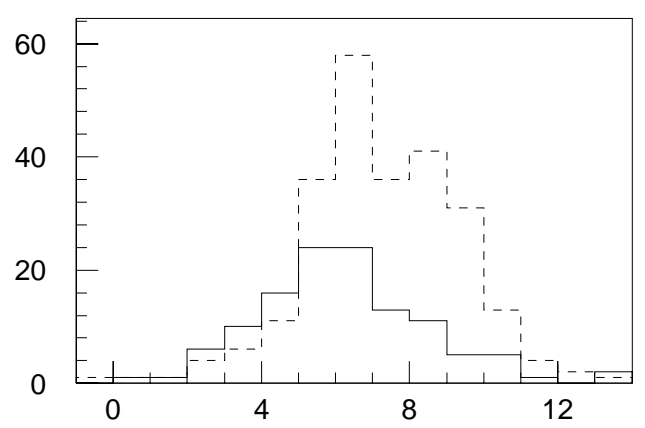

(g) Magnitude

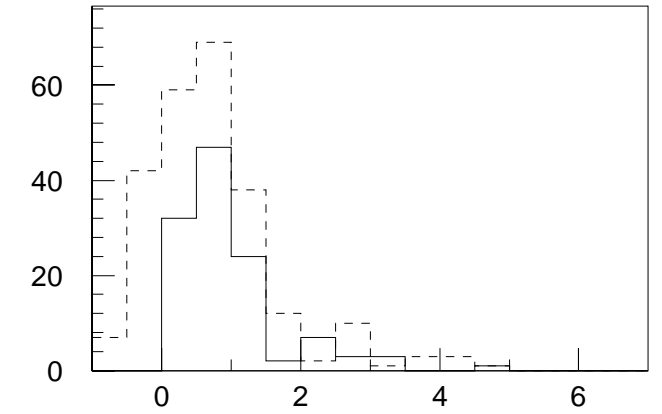

(b) Period (log days)

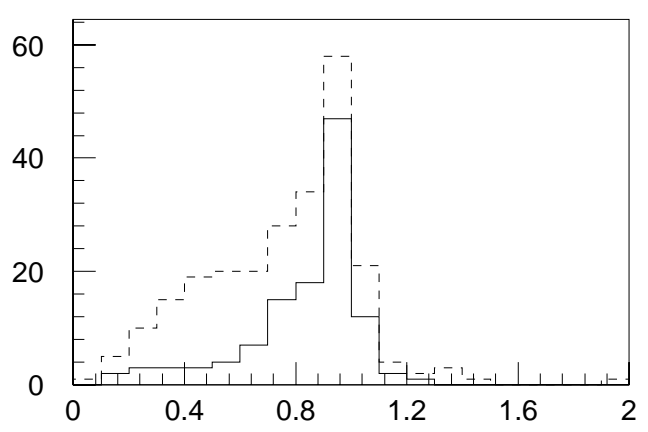

(d) Mass Ratio

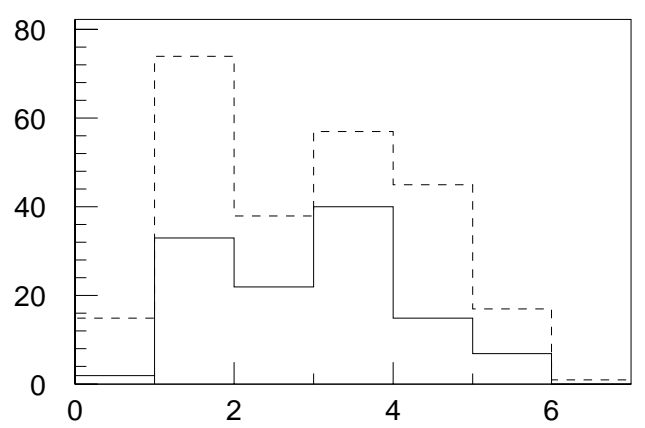

(f) Spectral Type

Figure 2.6: Histograms of 119 SBII systems in the Eighth DAO Catalogue with quality ratings $a$ or $b$ (solid lines), and $247 \mathrm{SBII}$ systems with quality ratings $c, d$, or $e$ (dashed lines). For a legenda to the histograms, see Table 2.3. 

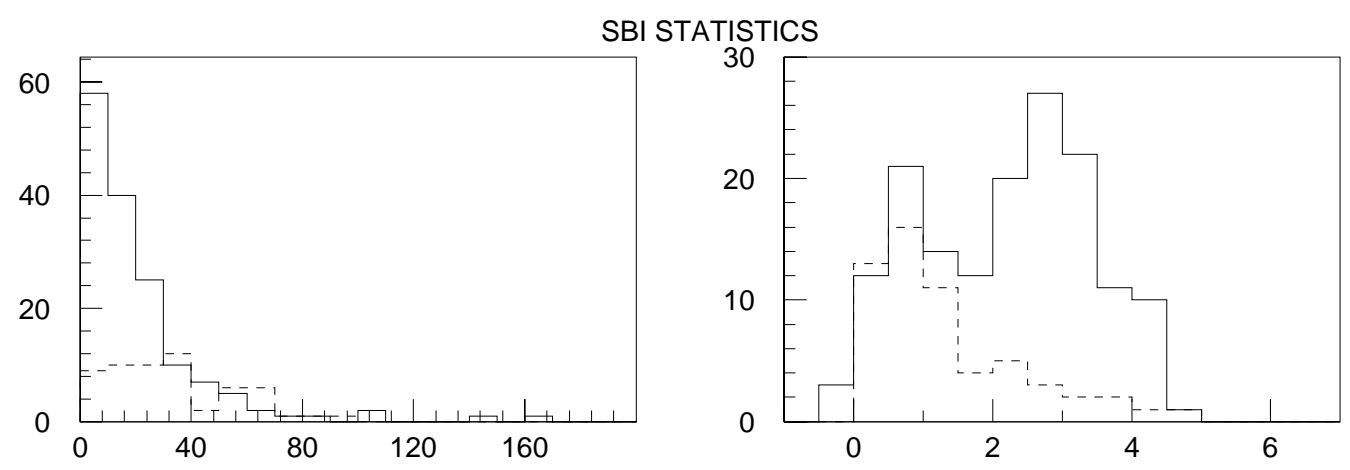

(a) $\mathrm{K} 1(\mathrm{~km} / \mathrm{sec})$

(b) Period (log days)

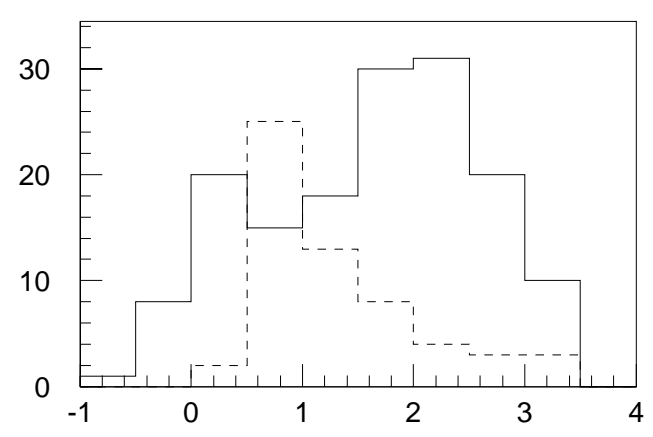

(c) Semi-Major Axis

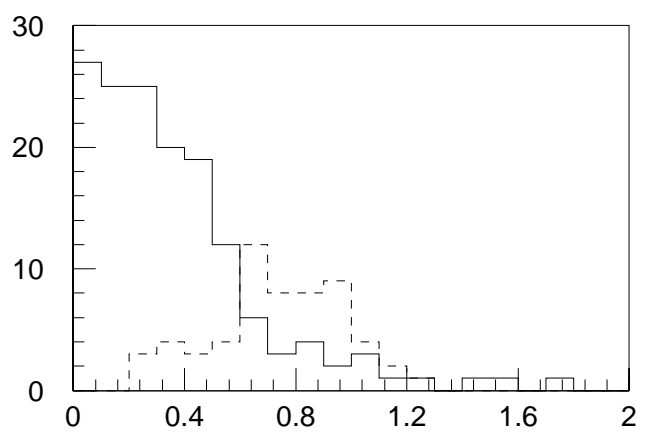

(d) Mass Ratio

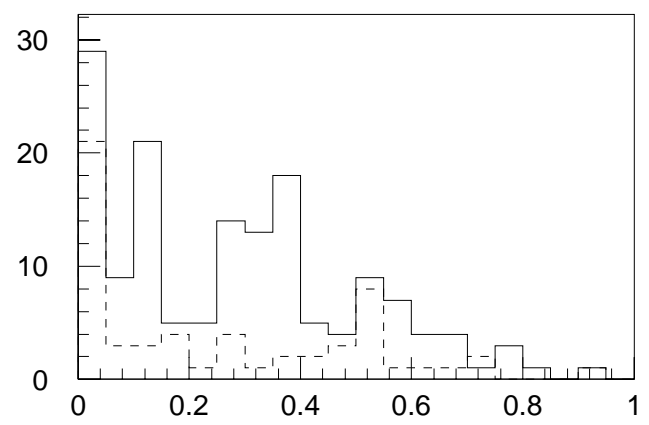

(e) Eccentricity

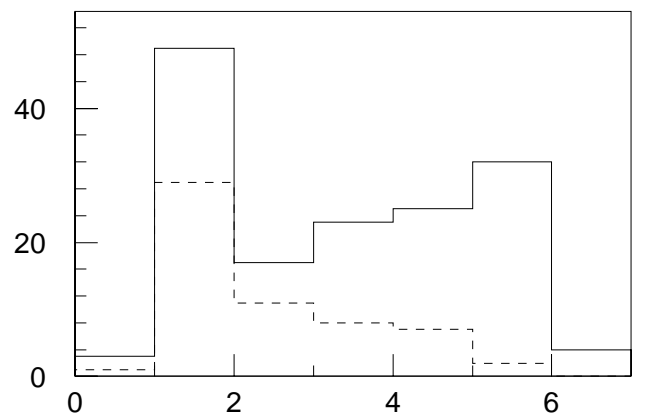

(f) Spectral Type

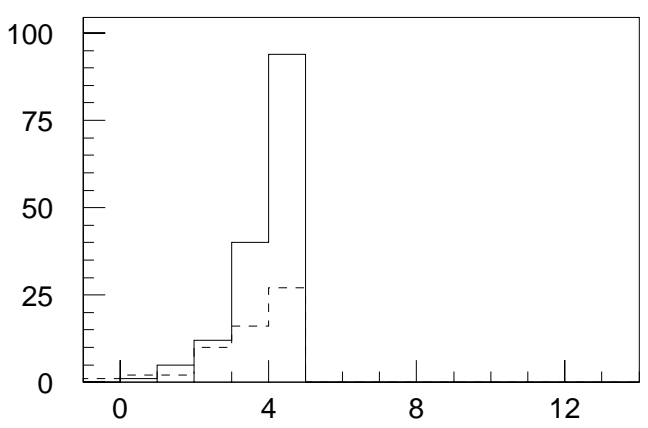

(g) Magnitude

Figure 2.7: Histograms of 153 SBI systems (solid lines), and 58 SBII systems (dashed lines) in the Eighth DAO Catalogue with magnitudes $m \leq 5^{\mathrm{m}}$. For a legenda to the histograms, see Table 2.3. 


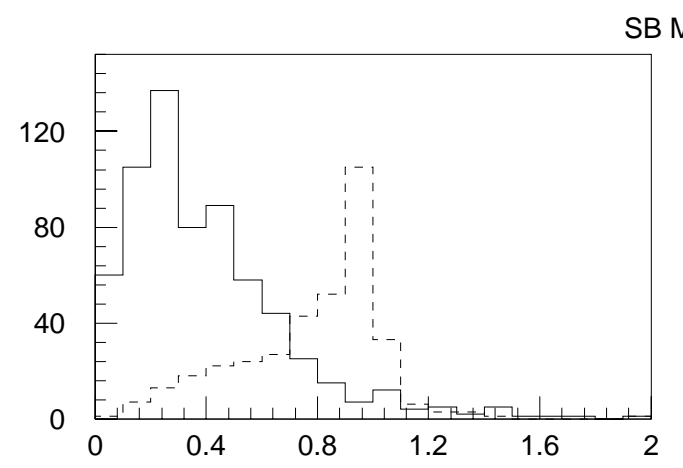

B Mass Ratios

ALL
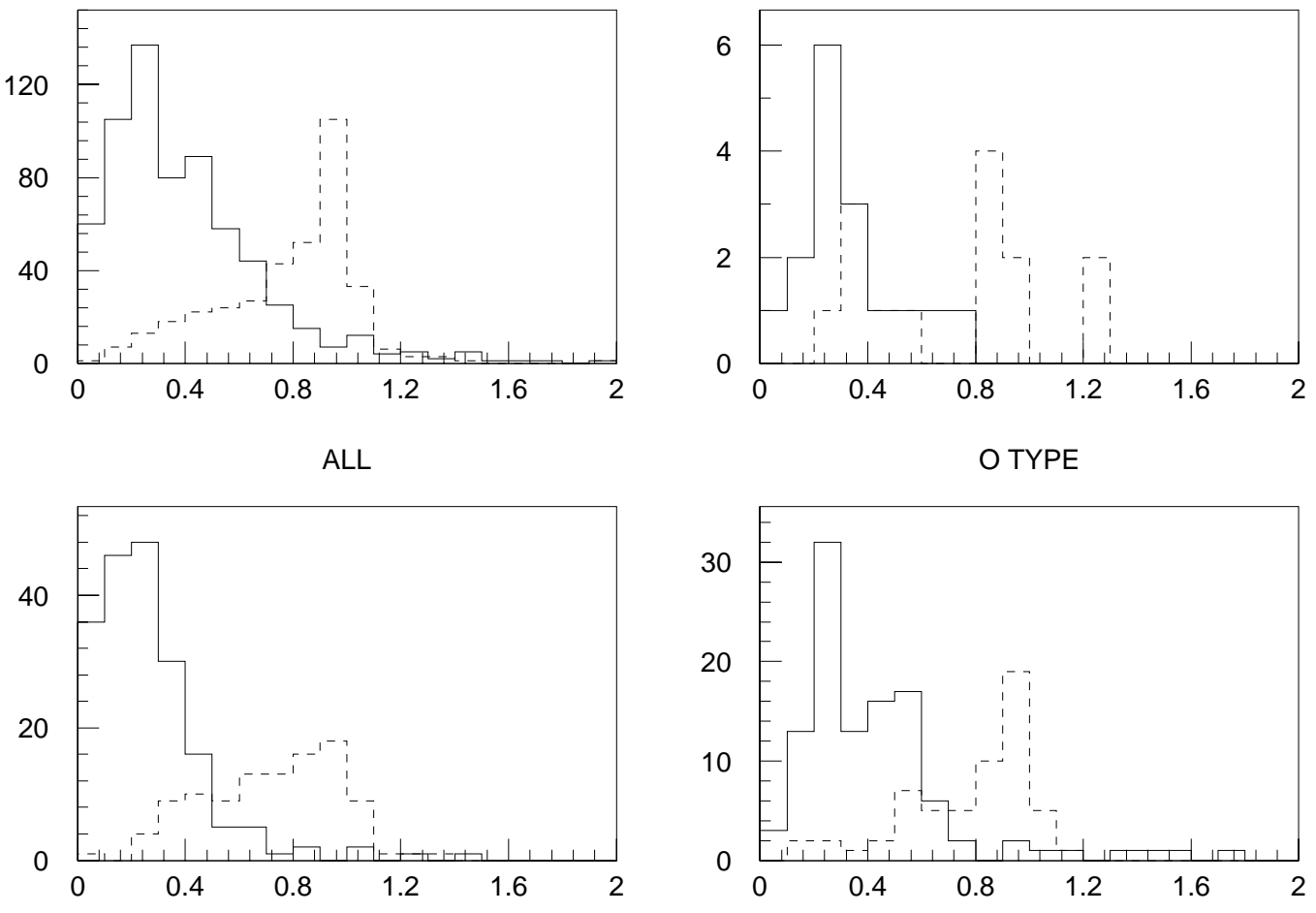

B TYPE
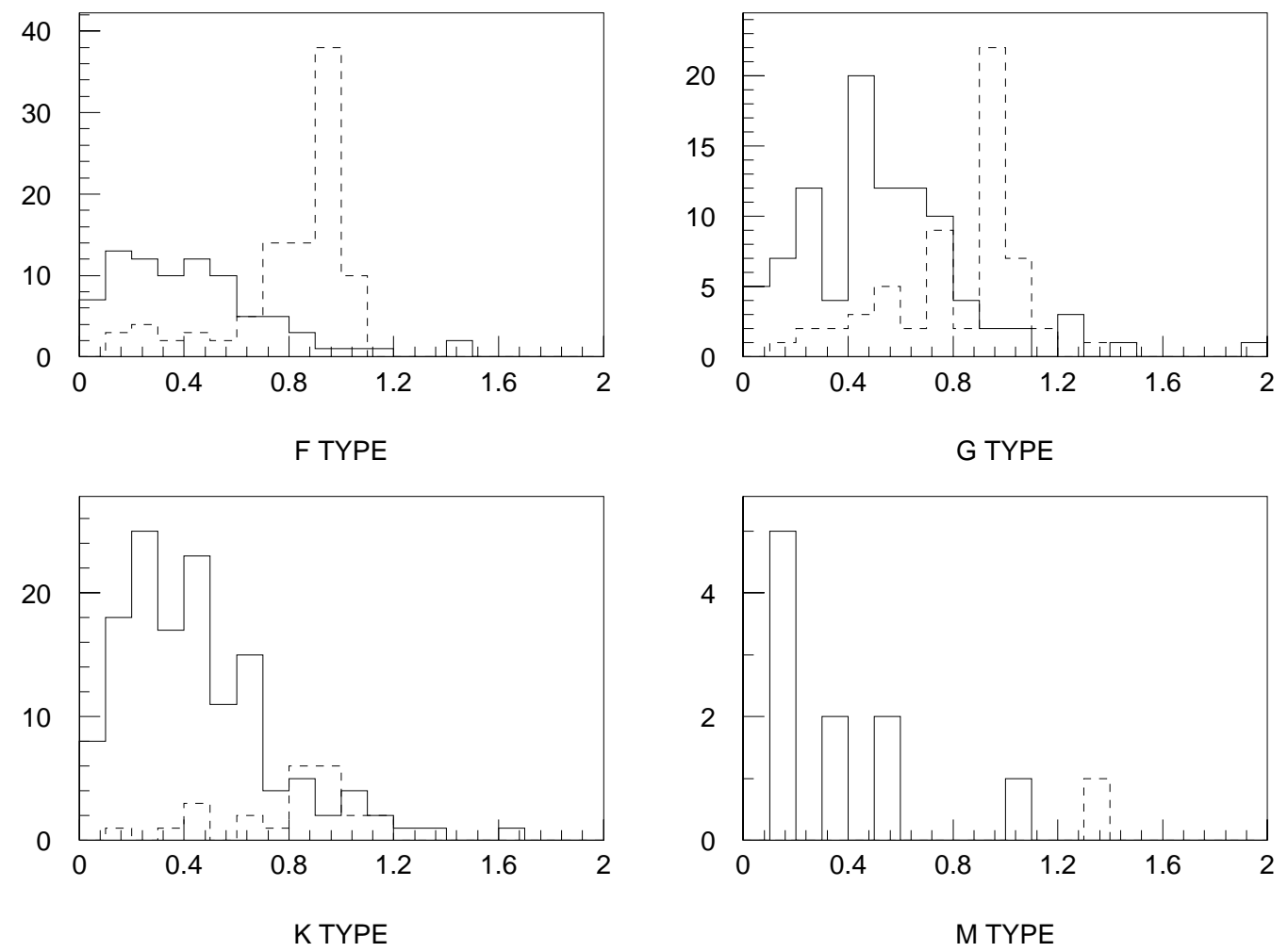

Figure 2.8: Histograms of the mass-ratio distributions of SBI (solid lines) and SBII (dashed lines) systems per spectral type in the Eighth DAO Catalogue. For the numbers of systems involved in the histograms, see Table 2.2. 

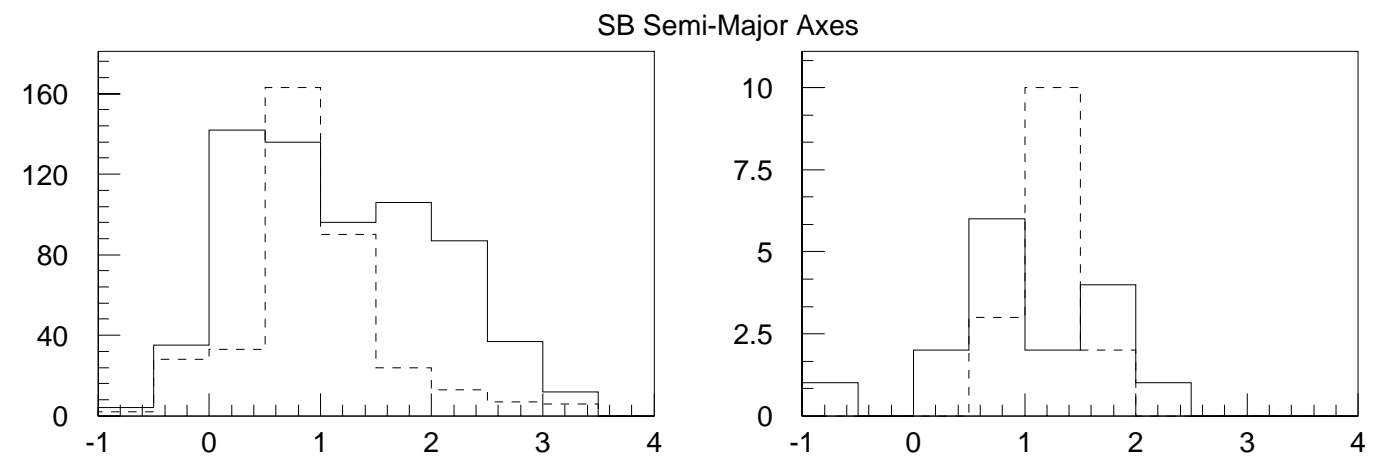

ALL
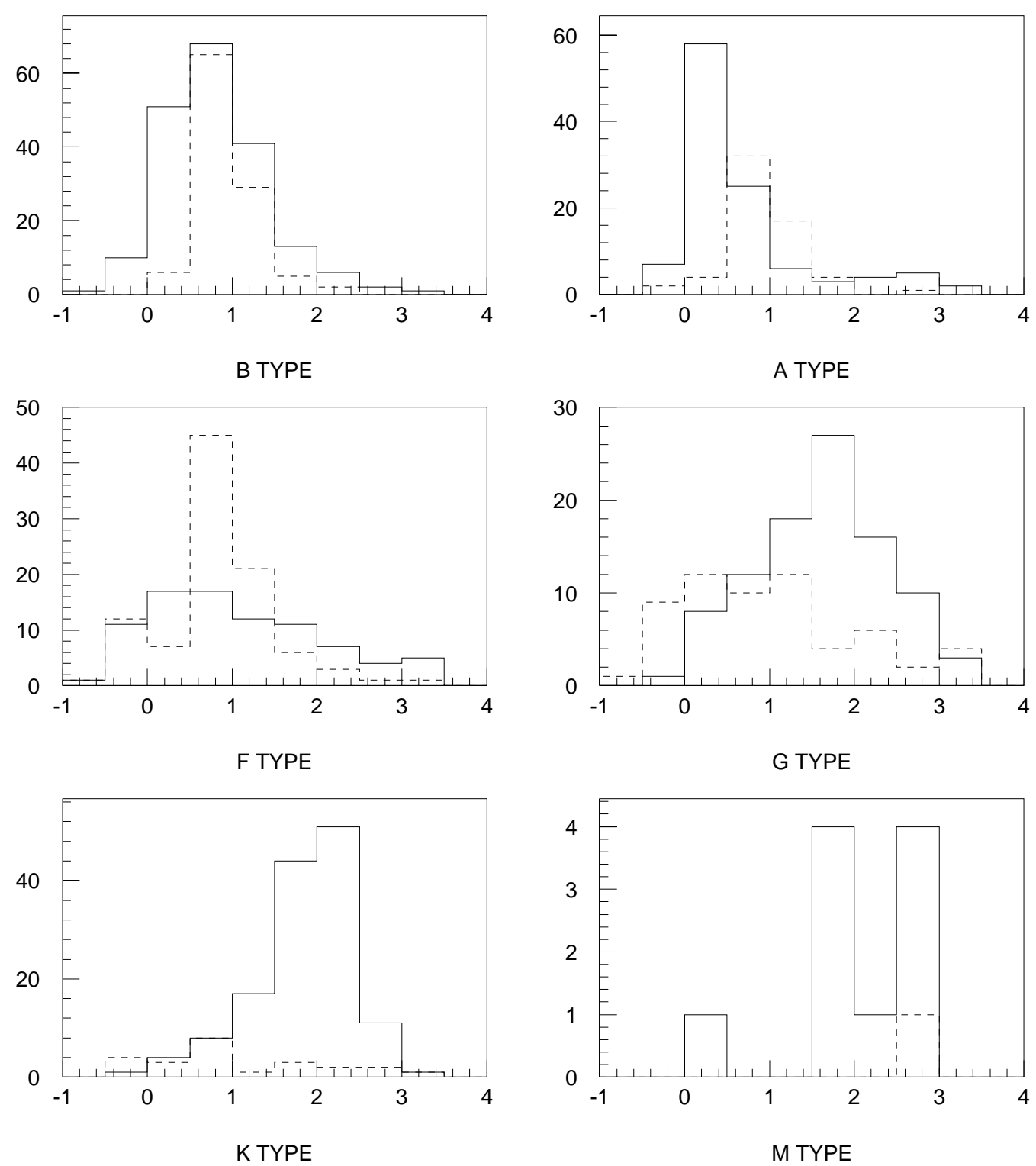

Figure 2.9: Histograms of the distributions of semi-major axes of SBI (solid lines) and SBII (dashed lines) systems per spectral type in the Eighth DAO Catalogue. Units are $\log \left(R_{\odot}\right)$. For the numbers of systems involved in the histograms, see Table 2.2. 


\section{Acknowledgements}

I wish to acknowledge stimulating discussions with Prof. E. P. J. van den Heuvel, and the valuable comments provided by Prof. J. van Paradijs. I thank Dr. J.-L. Halbwachs, of the Centre de Donnees Stellaires in Strasbourg, for providing me with a magnetic tape version of DAO7. I thank Drs. A. H. Batten and J. M. Fletcher, of the Dominion Astrophysical Observatory, Victoria, B.C., for providing me with a machine readable version of DAO8. My work is supported through the Netherlands Foundation for Research in Astronomy (Astron) by the Netherlands Foundation for the Advancement of Research (NWO), grant 782-371-026.

\section{References}

Abt, H. A.: 1979, Astron. J. 84, 1591

Abt, H. A.: 1983, Ann. Rev. Astron. Astrophys. 21, 343

Abt, H. A. and Levy, S. G.: 1976, Astrophys. J., Suppl. Ser. 30, 273

Abt, H. A. and Levy, S. G.: 1978, Astrophys. J., Suppl. Ser. 36, 241

Baranne, A., Mayor, M., and Poncet, J. L.: 1979, Vistas Astron. 23, 279

Batten, A. H.: 1967, Sixth Catalogue of the Orbital Elements of Spectroscopic Binary Stars, Dominion Astrophysical Observatory, Victoria, B.C., (DAO6)

Batten, A. H. and Fletcher, J. M.: 1989, Observatory 109, 186

Batten, A. H., Fletcher, J. M., and MacCarthy, D. G.: 1989, Eighth Catalogue of the Orbital Elements of Spectroscopic Binary Sytems, Dominion Astrophysical Observatory, Victoria, B.C., (DAO8)

Batten, A. H., Fletcher, J. M., and Mann, P. J.: 1978, Seventh Catalogue of the Orbital Elements of Spectroscopic Binary Sytems, Dominion Astrophysical Observatory, Victoria, B.C., (DAO7)

Fofi, M., Maceroni, C., Maravalle, M., and Paolicchi, P.: 1983, Astron. Astrophys. 124, 313

Garmany, C. D., Conti, P. S., and Massey, P.: 1980, Astron. J. 242, 1063

Griffin, R. F.: 1967, Observatory 148, 465

Griffin, R. F.: 1985, in P. P. Eggleton and J. E. Pringle (eds.), Interacting Binaries, pp 1-12, NATO ASI Series, D. Reidel, Dordrecht

Halbwachs, J. L.: 1987, Astron. Astrophys. 183, 234

Harmanec, P.: 1988, Bull. Astron. Inst. Czech. 39, 329

Hogeveen, S. J.: 1991, Astrophys. Space Sci., submitted

Jaschek, C. and Ferrer, O.: 1972, Publ. Astron. Soc. Pac. 84, 292

Kraicheva, Z. T., Popova, E. I., Tutukov, A. V., and Yungel'son, L. R.: 1978, Sov. Astron. 22, 670

Lucy, L. B. and Ricco, E.: 1979, Astron. J. 84, 401

Popova, E. I., Tutukov, A. V., and Yungelson, L. R.: 1982, Astrophys. Space Sci. 88, 55

Popper, D. M.: 1980, Ann. Rev. Astron. Astrophys. 18, 115

Sachs, L.: 1981, Applied Statistics, Springer-Verlag, New York

Staniucha, M.: 1979, Acta Astron. 29, 587

Trimble, V.: 1974, Astron. J. 79, 967

Trimble, V.: 1978, Observatory 98, 163

Trimble, V.: 1990, Mon. Not. R. Astron. Soc. 242, 79

Wolff, S. D.: 1978, Astrophys. J. 222, 556 